Artículo recibido:

31 de julio del 2012.

Evaluado:

16 de octubre del 2012.

Aceptado:

31 de octubre del 2012.

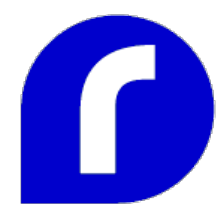

Daniel Cerdas Sandí

\section{Cultura y desarrollo: Tendencias hegemónicas y contra-hegemónicas}

RESUMEN

El trabajo busca presentar teorías o propuestas conceptuales sobre la relación cultura y desarrollo, pero no se limitará a una exposición de las mismas, sino que pretendemos realizar una crítica de la conceptualización de varias de estas nociones consideradas hegemónicas. Por otra parte, se presentará una propuesta alterna considerada más precisa y válida para entender la relación cultura y desarrollo en cuanto responde a un análisis propio de las estructuras socioeconómicas que condicionan dicho vínculo.

Palabras Clave

Cultura, desarrollo, hegemonía, ideología

\section{Culture and Development: Hegemonic and Counter - Hegemonic Trends}

\author{
ABSTRACT
}

This paper aims to present the theories or proposals about the relationship between culture and development but this does not limited the exposition of both of them, but the creation of a critical of the conceptualization of some of the notions considered as hegemonic. In the other hand, we present an alternative proposal considered as more accurate and valid for us to understand the relationship culture - development as the response to an analysis from the socioeconomics structures that determine that link.

KEY WORDS

Culture, development, hegemony,
Licenciado en Ciencias Políticas de la Universidad de Costa Rica (UCR) y profesor de esa misma universidad. El autor labora también en el Sindicato de Empleados del Banco Nacional (SEBANA) y la Confederación Costarricense de Trabajadores Democráticos (CCTD). Entre sus temas de interés están el desarrollo nacional, los regímenes de acumulación y los modos de regulación.

Correo electrónico: danielcerdas_3@yahoo.com 


\section{Cultura y desarrollo: Tendencias hegemónicas y contra-hegemónicas}

\section{Aclaraciones conceptuales previas}

En este trabajo se entenderá la cultura como lo definió E. B. Tylor en 1871: "Cultura, o civilización es todo complejo que incluye conocimientos, creencias, arte, leyes, moral, costumbres y cualquier otra capacidad o hábito adquiridos por el hombre [ser humano] en tanto miembro de una sociedad" (Payne, 2002, p. XVI). De esta forma, cultura será entendida en la relación ser humano-sociedad; todo aquello que se desprenda de la complejidad de la vida societal.

Por su parte, en cuanto a la definición de desarrollolograr dar una definición universalmente aceptada no es posible. Se trata de un concepto polisémico, además conflictivo, cuyo abordaje siempre invita al debate. Así las cosas, se presenta una definición bastante general que cumple las expectativas de este trabajo. El desarrollo aquí se entiende como desarrollo humano, según la definición que acoge el Programa de Naciones Unidas para el Desarrollo (PNUD). Se entiende por este aquel proceso por el cual una sociedad mejora las condiciones de vida de sus miembros a través de un incremento de los bienes y servicios con los que puede cubrir sus necesidades básicas y complementarias, y de la creación de un entorno en el que se respeten los derechos humanos de todos ellos. Lo anterior, de forma íntegra incluye aspectos relacionados al desarrollo social, al desarrollo económico (incluyendo el desarrollo local y rural) así como el desarrollo sostenible, además de la equidad de género. 
Se debe realizar también otra aclaración sobre lo comprendido por hegemonía. Este artículo parte del trabajo de Antonio Gramcsi (2004) para abordar este concepto. El autor italiano señalaba que las clases o fracciones de clase dominantes ejercían el poder no solo desde el uso legal de la violencia, ya sea desde toda la estructura de leyes o bien mediante el despliegue de fuerzas policiacas o militares, sino que otra manera de lograr perpetuarse en el poder y seguir manteniendo un sistema social acorde a sus intereses de clase es imponer una visión de mundo, una única cultura, es decir todo un sistema de ideas, costumbres, instituciones y valores. La reproducción y el mantenimiento de esa determinada cultura son esenciales para la reproducción del sistema mismo. Esa cultura reproductora del sistema es la que llamamos hegemónica o que ostenta hegemonía. Por tanto, la hegemonía va más allá de la gestión política de ejercer el poder y de tener acceso al uso legal de la violencia, sino que es también la conducción cultural de la sociedad. No solo hay hegemonía en la dirección política, social y económica, sino que también en la cultura.

Así, la discusión sobre el tema del desarrollo y la cultura está en si la cultura influye en las posibilidades de una sociedad de alcanzar el desarrollo. La cultura tiene un rol esencial, en cuanto engloba "conocimientos, creencias, arte, leyes, moral, costumbres y cualquier otra capacidad o hábito" de las personas que pretende llegar a cierto estadio social que se considere desarrollo.

De este modo, el asunto por abordar en este artículo es, en primera instancia, mostrar la conceptualización que algunos autores hacen de la relación cultura-desarrollo desde una determinada postura ideológica la cual en este documento se ha denominado hegemónica con el objetivo de evidenciar las debilidades de dichas propuestas. En segundo lugar, se busca mostrar la existencia de una concepción contra-hegemónica de la relación cultura-desarrollo, la cual es más pertinente para entender los procesos socioeconómicos.

\section{Cultura y desarrollo desde posiciones hegemónicas}

A continuación se exponen varias concepciones de la relación cultura-desarrollo. En primera instancia la propuesta de concebir la cultura como recurso, con lo cual deviene mostrar la cultura como objeto mercadeable. Posteriormente se muestra la concepción de la cultura como espacio de participación política y cómo esto conlleva a dar paso a oportunidades de desarrollo. En tercer lugar, se expone la propuesta de Bernardo Kliksberg, la cual menciona que los valores de la sociedad incidirán en la posibilidad de alcanzar el desarrollo. 


\section{La cultura como recurso}

La mercantilización de todo tipo de bienes, incluso los culturales, ha sido la tónica del industrialismo en especial desde su etapa fordista y, más aún, en la posfordista. Tanto así, que en algunas economías la cultura en general, más allá de la industria cultural, se contabiliza como parte importante del PIB (Achugar, 2000). Se debe aclarar que la relación entre cultura y economía no funciona igual en ambas vías. Es decir, mientras algunos productos con valor cultural generan valor económico, lo contrario -productos con valor económico que generan valor cultural- no siempre ocurre.

El mundo contemporáneo ofrece una visión muy distinta de lo que en otras épocas era la cultura, en especial antes de 1950 cuando era concebida como "bellas artes". Esa noción era contraria a los intereses mercantilistas. Así, por sus propias características entre ellas la baja rentabilidad, las "bellas artes" fueron asumidas por el Estado. Había una división entre "alta cultura" y cultura comercial. Hoy, la mercantilización ha llegado a todos los ámbitos culturales y ello ha implicado el retiro parcial del Estado en cuanto a políticas culturales (Achugar, 2000).

Para Achugar (2000), el papel del Estado en los temas culturales debe reinventarse, es decir no se trata de dejar el poder estatal al margen de la temática, ni tampoco que el Estado adquiera exclusividad en lo relacionado con la cultura, pues siempre se necesita del aporte de la sociedad. Lo que sugiere es un balance entre las industrias culturales y el Estado, el cual debe incidir en la democratización de la cultura mediante la ampliación del público receptor (Achugar, 2000).

Por otra parte, Yúdice (2002), desde una concepción cercana, indica que la cultura se ha expandido de una manera sin precedentes al ámbito político y económico, al tiempo que las nociones convencionales de cultura han sido considerablemente vacías y agrega además que tal vez sea más conveniente abordar el tema de la cultura en nuestra épocaconsiderándola como un recurso. Este autor señala que hay un uso creciente de la cultura como expediente para el mejoramiento tanto sociopolítico como económico, es decir, para la participación progresiva en esta época signada por compromisos políticos declinantes, conflictos sobre la ciudadanía y el surgimiento del capitalismo cultural.

Yúdice (2002) se pregunta el por qué del giro de la visión de la cultura donde esta se legitima según su utilidad. Esto lo responde indicando que "la globalización pluralizó los contactos entre los pueblos diversos y facilitó las migraciones, y de ese modo problematizó el uso de la cultura como expediente nacional" (Yúdice, 2002, p. 25), y agrega que al finalizarse la Guerra Fría la creencia en la libertad artística y con ello el apoyo incondicional a las artes se debilitó, pues era un apoyo motivado políticamente. 
En Estados Unidos, una vez acabada la Guerra Fría, se redujeron los gastos destinados a los temas culturales, al mismo tiempo los sectores de las artes y la cultura afirman que desde la cultura se pueden resolver los problemas norteamericanos: incrementar la educación, mitigar luchas raciales, crear empleos, reducir el delito y hasta generar ganancias. A estos se suman las posiciones que "le atribuyen al arte y la cultura múltiples propósitos: fomenta cohesión social en las políticas controvertidas, y contribuye a disminuir el desempleo. Esto llevó incluso a que el presidente del Banco Mundial, James $\mathrm{D}$, Wolfensohn, impulsara la inclusión de la cultura como catalizador del desarrollo humano" (Yúdice, 2002, pp. 26-27).

De esta forma, cada vez más se invoca la cultura "como motor del desarrollo capitalista, y ello se manifiesta en la repetición ad nauseam de que la industria audiovisual ocupa, en Estados Unidos, el segundo puesto después de la industria aeroespacial" (Yúdice, 2002, p. 31). Es importante agregar que este fenómeno de culturalización de la economía estadounidense no ocurrió naturalmente, sino que fue cuidadosamente coordinado mediante acuerdos sobre el comercio y la propiedad intelectual, tales como el GATT y la OMC, a través de leyes que controlan el movimiento intelectual y manual. En otras palabras, la nueva fase del crecimiento económico, la economía cultural, es también economía política. Incluso Yúdice (2002, p. 33) señala que "La culturalización es, además, economía política, pues el gobierno de Estados Unidos fue un factor central en garantizar que ese país pudiera mantener dominio de la nueva economía". Y continúa:

Pero esta culturalización no es para todos por igual, sino que recurrir a la creatividad económica evidentemente favorece a la clase profesional-gerencial por cuanto saca provecho de la retórica de la inclusión multicultural. Los grupos subordinados y minoritarios ocupan un lugar en este esquema en calidad de obreros no calificados que aportan servicios y en calidad de proveedores de vida étnica que aportan servicios y de otras experiencias culturales [...] que representan el nuevo estadio del desarrollo capitalista (Yúdice, 2002, p. 35).

Hasta aquí hemos mostrado una de las visiones de la relación cultura-desarrollo, la cual hace énfasis en las posibilidades que tiene la cultura como bien o servicio en la sociedad y a partir de la cual diversos sectores de la sociedad se pueden dedicar a esta como fuente de recursos que les permita acceder a una mejor vida, pero como lo señala Yúdice es necesaria la culturalización de la sociedad y hasta ahora esto se ha logrado de forma desigual. 
Por otro lado, esta concepción no aborda los problemas centrales que dan origen a la pobreza, la desigualdad y el subdesarrollo de los pueblos, contrario a esto solo expone las posibilidades que ofrece la cultura para mejorar las condiciones de vida de la sociedad pero no hace primero un análisis crítico de las circunstancias que han llevado a los seres humanos a convivir en condiciones deplorables, de ahí su principal debilidad como propuesta para hacerle frente a los problemas del desarrollo desde la cultura.

Además, no hay una discusión en relación con las características de la cultura, a los valores que se exponen en esas culturas o las implicaciones que conllevan determinas visiones de mundo. Hay, por tanto, implícito aquí una postura de que todo lo que sea cultural y comerciable es positivo, dejando de lado la cuestión ideológica de los conocimientos, creencias, arte, leyes, moral, costumbres y valores.

\section{Cultura, participación y dinámicas de desarrollo}

Hopenhayn (2000) se refiere a las oportunidades que ofrece la cultura como espacio de participación política, además de brindar mayores oportunidades en las dinámicas del desarrollo. Este autor parte de tres tesis: 1) Una modernización con capacidad creadora requiere de una ciudadanía moderna además de actores con capacidad de participar mediante un intercambio racional en el "mercado" político y en los espacios públicos, con verdaderas opciones de tener derechos en el plano social y en el jurídico, y con acceso a información y conocimientos para insertarse con mayores oportunidades productivas en la dinámica del desarrollo. 2) No puede limitarse el discurso sobre la ciudadanía a mecanismos representativos, especialmente en una región donde la falta de tolerancia y la resistencia a la diversidad cultural han sido base para discriminar entre ciudadanos de primera, segunda y tercera categoría. 3) La construcción de ciudadanía y la fuerza integradora de la modernidad desde la cuestión cultural tendrá que asumir dinámicamente dos grandes marcas de la historia cultural en la región: la dialéctica de la negación del otro como problema, y el tejido intercultural como potencia de la modernidad (Hopenhayn, 2000).

De esta forma son planteados dos grandes desafíos: primero, superar la larga tradición de la dialéctica de la negación del otro, en la cual la cultura discriminadora constituye el cimiento donde se monta una larga tradición de exclusión socioeconómica, cultural y sociopolítica. En segundo lugar, asumir positivamente nuestra condición de tejido intercultural, que es el conjunto de hibridaciones culturales que se producen por el cruce entre lo propio y lo externo. 
Sobre el primero, hay que tener presente que una cultura de la servidumbre y de la degradación sigue operando como eje de reproducción de la asimetría en muchos ámbitos de actividad económica y de relación social. Es necesario reconocer a los otros como parte de nuestra identidad colectiva . . Esto conduce al tejido intercultural, es decir a "asumirse en medio de una modernidad signada por una creciente "complejidad de la identidad»" (Hopenhayn, 2000 , p. 233). Por tanto, hay una transformación profunda de las relaciones simbólicas entre los diferentes grupos sociales. Se asiste también a un escenario en que los grupos locales se incorporan de manera acelerada a mercados simbólicos exógenos, lo que resulta en hibridación cultural.

Es imperioso señalar que esta disposición a combinar o mezclar culturas puede tener otro desenlace menos afortunado del deseado, pues más allá de pensar en la hibridación cultural, se puede dar en su lugar la imposición cultural, la aculturación o la pérdida total de alguna de las culturas, o bien una parte considerable de esa cultura.

Es importante agregar que el desarrollo de la industria cultural enfrenta en la región un gran reto en la medida en que puede constituirse en un poderoso resorte para la cultura participativa, una ciudadanía con vocación protagónica y actores socioculturales que se incorporen a la modernidad en el intercambio horizontal de símbolos y mensajes. Las posibilidades que hoy ofrece el complejo industrial cultural, en cuanto a los costos decrecientes y la flexibilidad para combinarse y articularse sistemáticamente Lo anterior le plantea a la sociedad un desafío de creatividad e inteligencia: se requiere desarrollar capacidad de inventiva y de adaptación, tanto desde la política cultural del Estado como entre los distintos actores económicos de la industria cultural. Lo anterior para capitalizar el potencial de participación social y cultural del nuevo complejo industrial cultural (Hopenhayn, 2000).

La propuesta de participación política, reconociendo las diferencias culturales de los diversos grupos sociales para lograr avances en desarrollo humano, es sugerente. Lo anterior en cuanto es válida la necesidad de avanzar al multiculturalismo y al reconocimiento de varias visiones de mundo, en especial de las concepciones no-occidentales y más aún de las poblaciones originarias. Salvo ese importante señalamiento esta propuesta, al igual que la primera que se ha mostrado, no se pregunta por las causas originarias del subdesarrollo y sus consecuencias negativas en las condiciones socioeconómicas de los pueblos. Además, esta conceptualización parte de una supuesta ausencia del conflicto en la sociedad tal como si las clases o fracciones de clases sociales no tuvieran visiones de mundo antagónicas. 


\section{Cultura para alcanzar el desarrollo}

Bernardo Kliksberg (2000) indica que, aunque hay avances en términos socioeconómicos con la globalización, muchas personas siguen en la miseria. Este autor también señala que la aldea global es compleja e incierta. Lo importante es que la idea del progreso indefinido está siendo sustituida por visiones que asignan un rol mayor a las complejidades, las contradicciones y las incertidumbres, que buscan soluciones a partir de la integración de perspectivas de análisis de la realidad, dejando de lado las clásicas concepciones que predican crecimiento económico como vía dedesarrollo humano(Kliksberg, 2000, p. 20).

Es por esto que la cultura en los procesos de desarrollo, en contraposición a nociones economicistas, son de gran importancia:

Hay múltiples aspectos en la cultura de cada pueblo que pueden favorecer a su desarrollo económico y social; es preciso descubrirlos, potenciarlos y apoyarse en ellos, y hacer esto con seriedad significa replantear la agenda de desarrollo de una manera que a la postre resultará más eficaz, porque tomará en cuenta potencialidades de las realidad que son de su esencia y que, hasta ahora, han sido generalmente ignoradas (Iglesias, 1997 citado en Kliksberg, 2000, pp. 20-21).

Hay una crisis en el pensamiento económico convencional. Así critica Kliksberg (2000) la orientación neoliberal de la economía, y señala que el desarrollo debe abordarse de manera integral, no como lo plantea la teoría del derrame, al enfocarse solo en el crecimiento económico. El ser humano no debe ser el medio sino el fin del desarrollo. Así las cosas, este autor considera el reenfoque profundo del pensamiento económico y ello se inscribe en la integración de los análisis del capital social y de la cultura. Es necesario atacar, al mismo tiempo desde esta perspectiva, los problemas económicos, los financieros, los sociales, y avanzar así en las transformaciones institucionales.

El capital social y la cultura son claves en esto para Kliksberg (2000). Acá el capital social es entendido como "el grado de confianza existente entre los actores sociales de una sociedad, las normas de comportamiento cívico practicadas y el nivel de asociatividad que caracteriza a esa sociedad" (Kliksberg, 2000 , p. 28). Promoviendo el capital social se logra disminuir los conflictos que surgen en las sociedades modernas. Sobre la cultura, indica que esta cruza todas las dimensiones del capital social de una sociedad. La cultura subyace tras los componentes básicos considerados capital social: confian- 
1. Los casos son: 1. Villa El Salvador, Perú, donde centenares de personas invadieron tierras y crearon una barriada con avances importantes. 2. Las ferias de consumo familiar de Venezuela donde se comercia a bajos precios. 3 . El Presupuesto Municipal de Porto Alegre donde hay una participación activa de los pobladores en la asignación del presupuesto municipal. za, comportamiento cívico y el grado de asociacionismo. Los valores de que es portadora una sociedad van a incidir en el desarrollo (Kliksberg, 2000).

Tres casos excepcionales ${ }^{1}$ valorados por Kliksberg (2000) muestran cómo el capital social y la cultura han ayudado a lograr mejores condiciones de vida en las poblaciones implicadas. Lo importante de estos tres ejemplos es que muestran cómo se logran considerables avances en términos de desarrollo humano y sus características principales son:

- $\quad$ Se basaron en la movilización de formas de capital no tradicional.

- Hubo adopción de un modelo de organización no tradicional en su totalidad.

- $\quad$ Papel importante de los valores: solidaridad, voluntarismo, civismo, entre otros.

A partir de dichas experiencias Kliksberg concluye que se debe de potencializar la cultura para movilizar el desarrollo, y ofrece tres ejes fundamentales para lograr esto:

1. Cultura y políticas sociales: utilización de saberes ancestrales y cultura popular, así mediante políticas dirigidas de la elevación de la autoestima grupal y personal de lo popular se fortalecerá la identidad colectiva y autoestima de la comunidad.

2. Cultura e integración social: Ante el problema de la exclusión, se debe de democratizar la cultura, de tal forma que se logre crear espacios asequibles a los excluidos con lo que se pueden hacer aportes efectivos en términos de pertenencia social y crecimiento personal.

3. Cultura y valores: Ante los valores dominantes como el individualismo, la indiferencia social, la falta de responsabilidad colectiva, el desinterés por el bienestar general, la búsqueda como valor central del enriquecimiento personal, el consumismo, entre otros, se debe promover una serie de valores que apunten en otra dirección como por ejemplo: la equidad, la solidaridad, la cooperación, la responsabilidad de los unos con los otros, el cuidado conjunto del bienestar colectivo, la superación de las discriminaciones, la erradicación de la corrupción, actitudes por el mejoramiento de la equidad, actitudes democráticas y el voluntarismo.

La propuesta que hace Kliksberg (2000) evidencia la importancia de atender las cuestiones culturales al momento de diseñar e implementar políticas públicas para el desarrollo humano, además que busca hacer partícipes activos del proceso de desarrollo a las personas. Sin embargo, a pesar de que el au- 
tor señala las deficiencias en el sistema actual, sigue ausente la discusión de las condiciones inherentes al sistema socioeconómico capitalista, con lo cual se deja a la cultura en una posición complicada. Aunque el autor haya indicado que es importante sumar las concepciones de mundo y los valores de los grupos sociales para avanzar hacia el desarrollo, la falta de interés en transformar la estructura socioeconómica socava las posibilidades de ese avance.

\section{Otra perspectiva de la relación cultura y desarrollo desde la contra-he- gemonía}

Hasta aquí se han mostrado algunas visiones sobre cómo entender la relación cultura y desarrollo, si bien estas presentan consideraciones plausibles a ser tomadas en cuenta, la dificultad de validarlas como propuestas radica en que no cuestionan las causas estructurales de los problemas del subdesarrollo en el sistema capitalista, es decir la acumulación de la riqueza en pocas manos, cuando esta es producida socialmente. Por esto, desde un inicio se ha indicado que dichas propuestas son hegemónicas, en cuanto no pretenden cambios sustanciales en la estructura socioeconómica capitalista, sino que son propuestas para gestionar los males resultantes del funcionamiento natural de esa estructura. Es por esto que se quiere exponer una visión alternativa.

Primero, se abordará el tema de la cultura como ideología. Es decir, un conjunto sistemático de creencias que intentan explicar al ser humano y al mundo, a la vez que orientan su conducta a partir de ciertos valores aceptados como correctos (Prado, 2010). En todas las sociedades encontramos teorías del mundo o ideologías puesto que, como señaló Engels en Ludwig Feuerbach y el fin de la filosofía clásica alemana, "todo lo que mueve a los hombres tiene que pasar necesariamente por sus cabezas" (1981, p. 387). Pero el marxismo, según Prado (2010), añade a este concepto general las siguientes peculiaridades. Las ideologías representan al ser humano y sus circunstancias en el mundo y la sociedad de una manera falseada. Esa deformación en la descripción del ser humano es consecuencia del interés de la clase dominante por conservarse como tal; como apuntaban Marx y Engels en La ideología alemana "las ideas de la clase dominante, son, en todas las épocas, las ideas dominantes" (1970, p.78).

La clase dominante dispone así tanto de los medios de producción material, como del control y producción de los bienes culturales, por lo que las representaciones que en una sociedad triunfen serán las que así disponga dicha clase social. Las ideologías son un "producto social": los pensamientos de los hombres y las mujeres son consecuencia de la sociedad en que están inmersos, en especial del orden económico que rija. 
De esta forma, Marx y Engels presentan el concepto de ideología como las representaciones que el ser humano se hace de la realidad ligada a las condiciones materiales objetivas:

Los hombres [los seres humanos] son los productores de sus representaciones, de sus ideas, etc., pero los hombres [los seres humanos] son reales y actuantes, tal y como se hallan condicionados por un determinado desarrollo de sus fuerzas productivas y por el intercambio que a él corresponde, hasta llegar a sus formaciones más amplias. La conciencia no puede ser nunca otra cosa que el ser consciente, y el ser de los hombres [los seres humanos] es su proceso de vida real. $\mathrm{Y}$ si en toda la ideología los hombres [los seres humanos] y sus relaciones aparecen invertidos como en la cámara oscura, este fenómeno responde a su proceso histórico de vida, como la inversión de los objetos al proyectarse sobre la retina responde a su proceso de vida directamente físico [...] La moral, la religión, la metafísica y cualquier otra ideología y las formas de conciencia que a ellas corresponden pierden, así, la apariencia de su propia sustantividad. No tienen su propia historia ni su propio desarrollo, sino que los hombres [los seres humanos] que desarrollan su producción material y su intercambio material cambian también, al cambiar esta realidad, su pensamiento y los productos de su pensamiento. No es la conciencia la que determina la vida, sino la vida la que determina la conciencia (Marx \& Engels, 1976, pp. 36-37).

Ahora bien, este aporte marxista sobre la cultura como ideología debe verse en relación con el tema del desarrollo, así las estrategias de desarrollo que son entendidas aquí como proyectos ideológicos que pretenden conducir lo económico, la política, lo social, lo ambiental y lo cultural, están estructurados sobre un considerable andamiaje de tipo técnico en donde el Estado aparece frecuentemente como un instrumento para la puesta en práctica de una determinada política económica y social (Kesselman, 1973, p. 11).

De lo anterior, pueden extraerse varias conclusiones. En primer lugar, toda estrategia de desarrollo debe ser entendida como un proyecto ideológico, es decir, como una serie de ideas, conceptos y visiones sobre cómo ha de ser. A 
su vez este conjunto determinará los mecanismos y la dirección del proceso mediante la estrategia hegemónica del momento, es decir la estrategia que goza de hegemonía. En tercer lugar, los proyectos ideológicos son sustentados y defendidos por diversas clases o fracciones de clase. Bourdieu (2000) señala que "Las diferentes clases y fracciones de clase están comprometidas en una lucha propiamente simbólica para imponer la definición del mundo social más conforme a su interés [...]" (p. 66).

Por su lado, Graciarena (1976) considera adecuado entender el desarrollo como estilo, definido como:

[...] un proceso dialéctico entre relaciones de poder y conflictos entre grupos y clases sociales, que derivan de las formas dominantes de acumulación de capital, de la estructura y tendencias de la distribución del ingreso, de la coyuntura histórica y la dependencia externa, así como de los valores e ideologías (p. 189).

Esta definición señala íntegramente el estilo de desarrollo. El concepto es amplio porque engloba diversas variables que chocan, convergen y se intersecan para finalmente definir la dirección del desarrollo y los mecanismos que harán posible el seguimiento de ese rumbo.

A diferencia de la propuesta de Kesselman (1973), para entender la conducción de la economía, la política y la sociedad, el concepto estilo de desarrollo según Graciarena (1976), es una estrategia donde "[...] una coalición de fuerzas sociales que imponen sus objetivos e intereses hasta que se agota por sus contradicciones implícitas" (p. 189).

El estilo de desarrollo se define por medio de la constante interacción entre las diversas fuerzas sociales que chocan o se complementan. Cada una sostiene determinadas estrategias de desarrollo o proyectos ideológicos específicos para conducir los diversos ámbitos de la organización política, económica y social. Esta interacción se condensa principalmente en el Estado, pues "en este contexto dialéctico se destaca particularmente la posición y el papel del Estado, su organización y función esenciales, y la de los grupos o élites que predominan en él e intentan establecer o preservar un estilo dado de desarrollo" (Graciarena, 1976, p. 190).

Al concepto de estilo de desarrollo es necesario agregar los aportes de Gramsci (2004). Por un lado este autor menciona que la hegemonía existe cuando se dan dos condiciones mínimas, por un lado la clase dominante tiene la capacidad o los recursos de poder para obligar a una clase social subordinada a que responda a sus intereses, lo cual implica una renuncia a su 
identidad y cultura común; pero además la clase dominante ejerce total control en las formas de relación y producción de todo el resto de la sociedad. Por otro lado, también se refiere a la hegemonía cultural como un proceso en el cual los subordinados debían imponer otro "escenario". En otras palabras, aunque se siga en la misma estructura social no debe verse como una conciliación entre clases al haber idealmente bases de contacto entre el ejercicio de la dirección política y el de la dirección intelectual (en una base social). Lo importante será avanzar, sumando cualquier tipo de alianza -interclasista de ser necesario- con el fin de conquistar un modelo cultural y contraponerlo al hegemónico (Gramsci, 2004, pp. 14-17, 276-283, 362-366).

En síntesis, al entender la cultura como ideología se denota que existe una cultura hegemónica y una o varias subalternas. Estas últimas responden, como menciona Kesselman (1973), a estrategias de desarrollo concretas, las cuales al entrar en contradicción dan como resultado un estilo de desarrollo. De esta forma no se ubica la cuestión del desarrollo de forma maniquea, sino como una constante lucha de distintas concepciones del mundo que están en fricción y que como producto de ese pugilato configuran el estilo de desarrollo.

La relación cultura y desarrollo no puede entenderse como se pretende desde ópticas hegemónicas en ausencia del conflicto socioeconómico. La aseveración de que la cultura impulsará el desarrollo, pero una cultura vaciada de contenido, supuestamente objetiva y sin rastros de proyectos de clase lleva únicamente a la perpetuación del sistema mismo, a la reproducción de la exclusión, la desigualdad y la pobreza con lo que no se garantizan avances concretos hacia el desarrollo humano, sino medidas de contención para no hacer el sistema socialmente insostenible.

\section{Consideraciones Finales}

La relación entre cultura y desarrollo, como se dijo al inicio, es compleja. Como paso decisivo es necesario dar la lucha desde las ideas, la lucha ideológica, entender que hay todo un entramado de ideas, costumbres, instituciones, leyes y valores que en ocasiones no son evidentes. Revelar esos proyectos ideológicos es necesario para la construcción de mejores herramientas conceptuales para alcanzar un acercamiento razonable a la realidad.

Es imprescindible, por tanto, tomar en cuenta la dimensión cultural para poder entender las dinámicas de desarrollo de determinadas sociedades, pero no se puede pasar por alto que la sociedad es heterogénea y por tanto allí tienen lugar tendencias hegemónicas y contra-hegemónicas, las cuales están en un constante devenir. En otras palabras, el proceso de desarrollo es diná- 
mico y no lo podemos analizar de manera estática, ni mucho menos estudiarlo únicamente en su dimensión económica. Esta lucha de proyectos ideológicos da la posibilidad de avanzar en términos de desarrollo humano que permitan la emancipación de los hombres y las mujeres, contraria a la lógica del sistema capitalista, pero esta no puede ser exitosa si la lucha emprendida desde sectores contra-hegemónicos no se articula con ejes culturales. Es en ese espacio desde donde las personas viven y entienden el mundo. Ignorar lo anterior hace que cualquier proyecto de nueva sociedad vaya al fracaso. La superación definitiva de las ideologías solo podrá realizarse con la desaparición de la explotación del ser humano por el ser humano.

\section{Bibliografía}

Achugar, H. (2000). Desafíos económico-culturales de América Latina (cultura "tradicional" e industrias culturales). En B. Kliksberg \& L. Tomassini (Eds.). Capital social y cultura: claves estratégicas para el desarrollo México D.F.: BID-FCE.

Bourdieu, P. (2000). Intelectuales, política y poder. Buenos Aires: Eudeba.

Engels, F. (1981). Ludwig Feuerbach y el fin de la filosofía clásica alemana. En C. Marx. \& F. Engels. Obras Escogidas. Moscú: Progreso.

Graciarena, J. (1976). Poder y estilo de desarrollo. Revista de la CEPAL, 1(1), 173-193.

Gramsci, A. (2004). Antología. Buenos Aires: Siglo XXI.

Hopenhayn, M. (2000). Cultura y participación: entradas para el debate. En. B. Kliksberg \& L.

Iglesias, E. V. (1997). Cultura, educación y desarrollo. Exposición en ocasión de la Asamblea General de la UNESCO, París.

Kesselman, R. (1973). Las Estrategias de desarrollo como ideologías. Buenos Aires: Siglo XXI Argentina Editores.

Kliksberg, B. (2000) El rol del capital social y de la cultura en el proceso de desarrollo. En. B. Kliksberg \& L. Tomassini (Eds.). Capital social y cultura: claves estratégicas para el desarrollo México D.F.: BID-FCE. 
Marx, K. \& Engels, F. (1976). La ideología alemana. México D.F.: Ediciones de Cultura Popular.

Payne, M. (2002). Diccionario de teoría crítica y estudios culturales. Buenos Aires: Paidós.

Prado, S. (2010). Teoría de la Ideología. Marxismo e Ideología. Recuperado de http://samuelpradof.blogspot.com/2010/01/articulo-teoria-de-la-ideologia. html

Yúdice, G. (2002). El recurso de la cultura. Barcelona: GEDISA. 